\title{
Can quantitative MRI be used in the clinical setting to quantify the impact of intra- articular glucocorticoid injection on synovial disease activity in juvenile idiopathic arthritis?
}

Joshua L. Bennett ${ }^{1}$, Amanda Wood ${ }^{1}$, Nicola Smith², Ravi Mistry ${ }^{3}$, Karen Allen', Sharmila Jandial', John D. Tuckett ${ }^{4}$, S. Claire Gowdy ${ }^{5}$, Helen E. Foster ${ }^{1,2}$, Flora McErlane ${ }^{1,2+}$ and Kieren G. Hollingsworth ${ }^{6^{* \dagger}}$ (D)

\begin{abstract}
Background: Juvenile idiopathic arthritis (JIA), the most common chronic rheumatic disease of childhood, is characterised by synovitis. Clinical assessments of synovitis are imperfect, relying on composite and indirect measures of disease activity including clinician-reported measures, patient-reported measures and blood markers. Contrast-enhanced MRI is a more sensitive synovitis assessment technique but clinical utility is currently limited by availability and inter-observer variation. Improved quantitative MRI techniques may enable future development of more stringent MRI-defined remission criteria.

The objective of this study was to determine the utility and feasibility of quantitative MRI measurement of synovial volume and vascularity in JIA before and twelve weeks after intra-articular glucocorticoid injection (IAGI) of the knee and to assess the acceptability of MRI to participating families.
\end{abstract}

Methods: Children and young people with JIA and a new episode of knee synovitis requiring IAGI were recruited from the Great North Children's Hospital in Newcastle upon Tyne. Quantitative contrast-enhanced MRI was performed prior to and twelve weeks after IAGI, in addition to standard clinical assessment tools, including the three-variable clinical juvenile arthritis disease activity score (CJADAS) and active joint count.

Results: Eleven young people (5 male, median age 13 years, range 7-16) with JIA knee flare were recruited and 10 completed follow-up assessment. Following IAGI, the median (interquartile range) CJADAS improved from 8.5 (2.7) to 1.6 (3.9), whilst the median synovial volume improved from $38.5 \mathrm{~cm}^{3}\left(82.1 \mathrm{~cm}^{3}\right)$ to $0.0 \mathrm{~cm}^{3}\left(0.2 \mathrm{~cm}^{3}\right)$. Six patients presented with frank synovitis outside normal limits on routine MRI reporting. A further three had baseline MRI reports within normal limits but the quantitative measurements identified measurable synovial uptake. Post-IAGI quantitative measurements highlighted significant improvements in 9 patients.

(Continued on next page)

\footnotetext{
* Correspondence: kieren.hollingsworth@newcastle.ac.uk

†Flora McErlane and Kieren G. Hollingsworth contributed equally to this

work.

${ }^{6}$ Newcastle Magnetic Resonance Centre, Institute of Cellular Medicine,

Newcastle University, Newcastle upon Tyne, UK

Full list of author information is available at the end of the article
}

(c) The Author(s). 2019 Open Access This article is distributed under the terms of the Creative Commons Attribution 4.0 International License (http://creativecommons.org/licenses/by/4.0/), which permits unrestricted use, distribution, and reproduction in any medium, provided you give appropriate credit to the original author(s) and the source, provide a link to the Creative Commons license, and indicate if changes were made. The Creative Commons Public Domain Dedication waiver (http://creativecommons.org/publicdomain/zero/1.0/) applies to the data made available in this article, unless otherwise stated. 
(Continued from previous page)

Conclusions: IAGI led to a marked reduction in synovial volume, with quantitative MRI identifying more patients with an improved synovial volume than routine qualitative clinical reporting. Improvements in cJADAS scores were more variable with the patient/parent global assessment component contributing most to the scores. Further work is indicated, exploring the utility of quantitative MRI in the assessment of less accessible joints and comparing the impact of different treatment modalities.

Keywords: Juvenile idiopathic arthritis, Disease activity assessment, Quantitative MRI, Synovitis, Synovial volume, Intra-articular glucocorticoid injection, Remission

\section{Background}

Juvenile idiopathic arthritis (JIA) is an umbrella term, summarising the International League of Associations for Rheumatology (ILAR) classification system for the markedly heterogeneous chronic idiopathic paediatric arthritides $[1,2]$. JIA is the most common chronic rheumatic disease in children and young people with a UK prevalence of 1:1000 [3]. Affected joints are characterised by synovial proliferation and inflammatory cell infiltration, resulting in synovial effusion and hypertrophy.

JIA is a relapsing and remitting condition and the majority of young people will have more than one inflammatory flare during the two years following initial diagnosis. At least onethird of young people continue to have episodes of active inflammation during their adult years [4]. Long-standing poorly controlled synovitis is associated with a high frequency of joint damage and joint replacement surgery in adults with JIA [5]. Early aggressive therapy, including intraarticular glucocorticoid injection (IAGI) and prompt introduction of systemic immunosuppression, is thought to improve remission rates, prevent joint damage and improve functional outcomes in JIA [6]. IAGI induces rapid suppression of inflammation in targeted joints through a complex combination of effects including reduced leucocyte cell infiltration, altered leucocyte activity, reduced synovial perfusion and reduced vascular permeability $[7,8]$.

The heterogeneous nature of JIA and normal developmental changes of childhood ensure that no single clinical assessment can reliably capture overall disease activity in all young people with JIA [9]. At the present time, clinicians rely on composite measures of disease activity, comprising multiple indirect clinical and blood markers, including the active and limited joint counts (AJC / LJC), disability score (CHAQ) [10], ESR/CRP and global assessments by clinicians and parents. None of these accurately reflects the synovial state, and many are subjective in nature with limited repeatability [11]. JIAspecific composite tools have been used to derive multiple definitions of remission and it is not yet clear which, if any, constitutes the optimal definition [12]. The lack of a single 'gold standard' treatment target is one of the central barriers to implementing modern treat-to-target regimes in clinical practice [13].
Newer imaging modalities, such as musculoskeletal ultrasound (MSUS) and magnetic resonance imaging (MRI), have the potential to further inform our understanding of remission in JIA. Comparison of clinical and imaging-based definitions of remission is considered a high priority for future research [14].

Ultrasound (US) and colour Doppler techniques can detect subclinical synovitis in young people with JIA, and there have been extensive efforts in recent years to define the normal US appearance of paediatric joints [15], to create definitions for the sonographic features of synovitis in young people [16], and to standardise acquisition protocols and scoring systems [17]. Although this work is important, paediatric MSUS requires specialist skills and training and may not be available at all centres. Furthermore, the non-uniform accessibility of many joint spaces limits MSUS scoring to broad grades [18].

Contrast-enhanced MRI is able to visualise the synovitis that drives the degradative disease process, even at low levels, and is recognised to be the most sensitive technique for the assessment of synovitis [19-21]. Postcontrast T1-weighted images clearly differentiate the extent of synovial hypertrophy vs. free fluid in the joint. In fact, MRI can identify synovial changes indicative of active disease in young people with JIA who fulfil clinical criteria for inactive disease [22]. Unenhanced MRI can uniquely detect bone oedema in addition to bone erosions. However, the clinical utility of MRI has been modest due to availability and the acceptability of MRI scanning in paediatric populations. While there has been research activity demonstrating the potential of MRI in the assessment of intra-articular disease activity, there are limited normative paediatric data, recruited cohorts have had mixed presentations and there is a lack of JIAspecific clinical assessment tools to validate the findings. Consequently, there is wide inter-observer variation in the interpretation and reporting of MRI scans.

In clinical practice, synovial enhancement is reported as either being within or exceeding normal limits, based on an expected normal appearance. In research, the qualitative JAMRIS score (juvenile arthritis MRI scoring system) has been proposed as a classification of synovial hypertrophy on a 3 -point scale $(<2 \mathrm{~mm}, 2-4 \mathrm{~mm}$ or $>4$ 
$\mathrm{mm}$ thickness) in six anatomical regions but may be insensitive to longitudinal change [23, 24]. By contrast, we can seek to quantify properties of disease activity on continuous scales by image processing, rather than qualitative description or grading. The potential advantage of such an approach is the ability to discriminate change more finely than qualitative scales, and to measure disease activity that may fall below present qualitative reporting standards [25-27]. The cost of such approaches is the analysis effort required.

In summary, quantitative MRI may have considerable utility in both clinical research and the clinical care of young people with JIA, since it can provide sensitive, continuous measurement of synovial volume and the vascularity of the synovium through dynamic measurement of the uptake of gadolinium contrast agent. This study investigates whether quantitative MRI can provide a sensitive measurement of the effects of IAGI in young people with JIA, and compares synovial volume and contrast uptake measurements. The study also assesses the feasibility and acceptability of the imaging for children and parents through feedback by questionnaire and telephone interviews.

\section{Methods}

\section{Subjects}

Young people were recruited from a tertiary paediatric rheumatology service in the North of England (The Great North Children's Hospital at Newcastle upon Tyne Hospitals NHS Foundation Trust). Patients and their families attending clinic were invited to consider participating in the study if all inclusion criteria were present (new or known diagnosis of JIA, new presentation of knee synovitis requiring intra-articular glucocorticoid injection (IAGI) and age 4-16 years). Exclusion criteria were steroid injection of involved knee in the past six months, concurrent use of oral or IV steroids, contraindications to contrast agent or non-sedated MRI, non-English speaking families, or recent trauma to the knee. No changes were made to concomitant immunosuppressive treatments during the study period. Families of young people who were eligible and interested at their clinic visit were followed up by telephone and invited to take part in the study.

Additional file 1: Fig. S1 shows the recruitment flow chart for the study. The subjects had two MRI and clinical assessments, the first prior to the intra-articular injection and the second at least twelve weeks after the injection. Affected knees were injected with $1 \mathrm{mg} / \mathrm{kg}$ triamcinolone hexacetonide to a maximum of 40 or $60 \mathrm{mg}$ depending on weight: image guidance was not used, as per local protocol. Where both knees were affected, both were injected, and the knee judged most affected at clinical examination was followed by MRI. The study, performed between September 2017 and August 2018, complied with the Declaration of Helsinki and obtained a favourable opinion from the Newcastle and North Tyneside 1 Research Ethics Committee and the Health Research Authority, with the parents/carers of the subjects giving written informed consent, and written assent from the young people. Routine clinical care was not altered by the addition of the MRI scanning. KGH had control of the study data and had responsibility for overseeing data acquisition and processing.

\section{Clinical assessments}

Clinical assessment was performed at baseline and follow-up. This included measurement of the core outcome variables: binary 74-joint active and limited joint counts, physician global score of disease activity $(10 \mathrm{~cm}$ visual analogue scale (VAS)), patient/parent global score of disease activity (10 cm VAS), CHAQ and ESR/CRP (where available) [28], a global pain score $(10 \mathrm{~cm}$ VAS) and the 3 and 4 variable juvenile arthritis disease activity scores (cJADAS and JADAS) [29, 30]. Clinical remission was defined according to the JADAS and cJADAS cutoffs for inactive disease $(</=1)$ [31, 32].

\section{MRI protocol}

A peripheral venous cannula was inserted in the upper limb. Subjects were made comfortable in a supine feet-first orientation on a Siemens 1.5 T Espree, using an extremity birdcage coil. The MRI sequences (including T2-, T1- and proton density weighted scans, Additional file 1: Table S1) were prescribed so that the bottom slice passed through the proximal aspect of the superior tibiofibular joint. The leg position and anatomical location were carefully matched at the post-treatment visit. The sequences included pre- and post-contrast T1-weighted (T1w) imaging to distinguish synovial enhancement and a multiple dynamic T1w sequence was used to collect dynamic gadolinium uptake data before, during and after the injection of contrast agent. After two dynamics (26s), the contrast agent was administered by hand as a standard single dose of gadoterate meglumine (Dotarem, $0.2 \mathrm{ml} / \mathrm{kg}$ body weight, Guerbet), followed by a $3 \mathrm{ml}$ flush of $0.9 \%$ sodium chloride. The uptake of contrast was imaged for five minutes postinjection. The post-contrast T1w fat suppressed turbo spin echo imaging was collected immediately after the dynamic series ended such that post-contrast data were collected 5 min post injection as recommended [33].

\section{Image processing}

The MRI images were reported by a consultant radiologist (JDT) and were also quantitatively processed using custom software in MATLAB 2017a (Mathworks, UK) and ImageJ $\mathrm{v} 1.43 \mathrm{u}(\mathrm{NIH}, \mathrm{USA})$. The images of the dynamic gradient 
echo sequence and the post-contrast T1w sequence were registered to the pre-contrast $\mathrm{T} 1 \mathrm{w}$ sequence to eliminate the effect of minor subject movement during the scan. A difference image stack was formed by subtracting the image intensity of the pre-contrast T1w sequence (Fig. 1a) from the post-contrast (Fig. 1b) to highlight the intensity of gadolinium uptake (Fig. 1c). This difference image was thresholded and binary gated to allow semi-automated delineation of the enhancing synovium from adjacent tissues throughout the imaging volume (Fig. 1d): the synovial volume was calculated from this by automatically summing the areas from each slice and multiplying by the slice thickness (see Additional file 1: for further detail). The signal increase due to contrast agent entering the synovium was expressed as a percentage increase compared to the first four dynamics before the gadolinium arrived (Fig. 2). Using the delineation of the synovium, the initial rate of uptake was estimated (in \%/s) from the gradient of the first two measurements after the arrival of contrast (Fig. 2). The signal enhancement (in \%) was calculated as the mean percentage increase of the final four dynamics of the series (Fig. 2, see Additional file 1 for further detail). Quantitative analysis was performed by two observers working independently (KGH, an MRI physicist with 12 years' experience in MSK imaging, and JLB, a specialist trainee in paediatrics).

\section{Patient acceptability questionnaires and telephone feedback}

To assess the acceptability of our research protocol, we asked the families to provide feedback in two ways. At the end of the first MRI appointment, young people and their families were invited to complete a NHS England 'Friends and Family' questionnaire [34] and this was followed up with a telephone interview in which the families were asked to discuss their experience of the MRI visit in more detail. The topics presented in this telephone interview and the formal analysis methods used are given in Additional file 1.

\section{Time occupation of the scanner suite, and study subject time}

For all MRI sessions, scan timings were extracted from the DICOM headers. Where feasible, the total time between the families arriving and leaving the MRI unit was measured.

\section{Statistical analysis}

Data were analysed in SPSS version 24 (RSI, Boulder) and expressed as median (interquartile range, IQR) except where medians and ranges have been explicitly stated. The changes in synovial volume, initial rate of contrast uptake and signal enhancement between the baseline and follow-up visits, AJC, cJADAS and pain

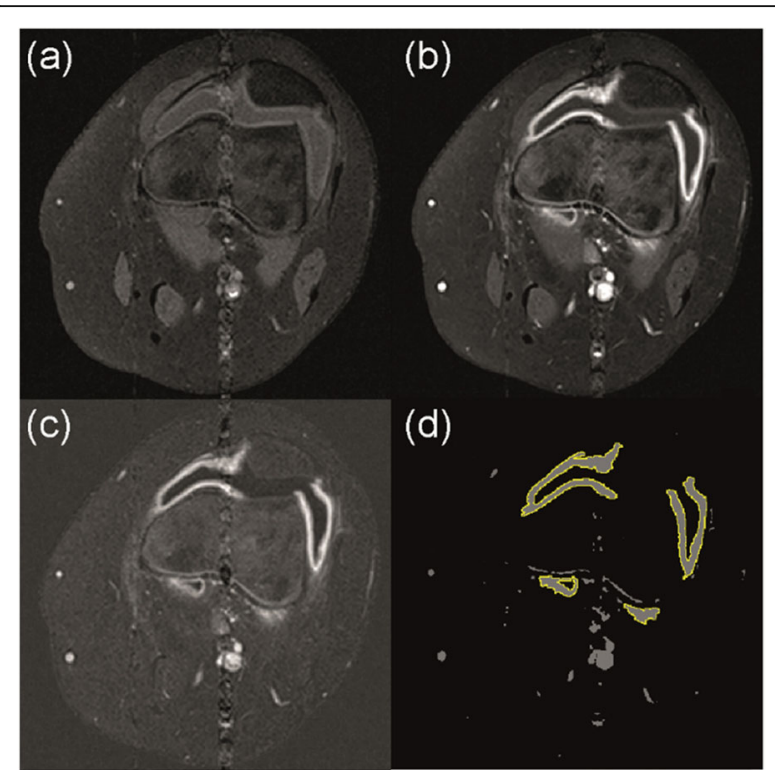

Fig. 1 Procedure for calculating the synovial volume, shown on one slice of the imaged knee volume. The acquired data are (a) the multi-slice T1-weighted FS TSE pre-contrast and (b) post-contrast showing synovial hypertrophy. (c) These image stacks are subtracted to produce a stack of difference images which highlight the signal change caused by contrast uptake compared to other tissues; (d) thresholding these values helps to segment the enhancing synovium from structures with low uptake, though blood vessels remain visible. The magic wand tool of ImageJ is used to select the enhancing synovium; the volume is calculated by summing across the image stack. FS TSE, fat saturated turbo spin echo

global score were analysed with the non-parametric Wilcoxon signed-rank test. The inter-observer agreement was assessed by using Bland-Altman analysis [35]. The bias and $95 \%$ limits of agreement was calculated between the two observers. Association between synovial volumes and clinical assessments was performed using the Spearman's rank test.

\section{Results}

Eleven young people ( 5 male; 6 female; median age 13.4 years; age range 7.7-16.0) participated successfully in the baseline studies. Demographic and clinical data (including concomitant medication) are presented in Table 1. Ten young people attended and successfully completed the second MRI research visits: contact with one family was lost and follow-up data could not be collected. Only one family approached with study information declined to take part in the research.

The median time between initial clinic visit and first MRI assessment was 7 days (range 6-8 days). IAGI was usually performed later the same day (median 0 days after initial MRI, range 0-21 days). The median time from joint injection to the second MRI and clinical 


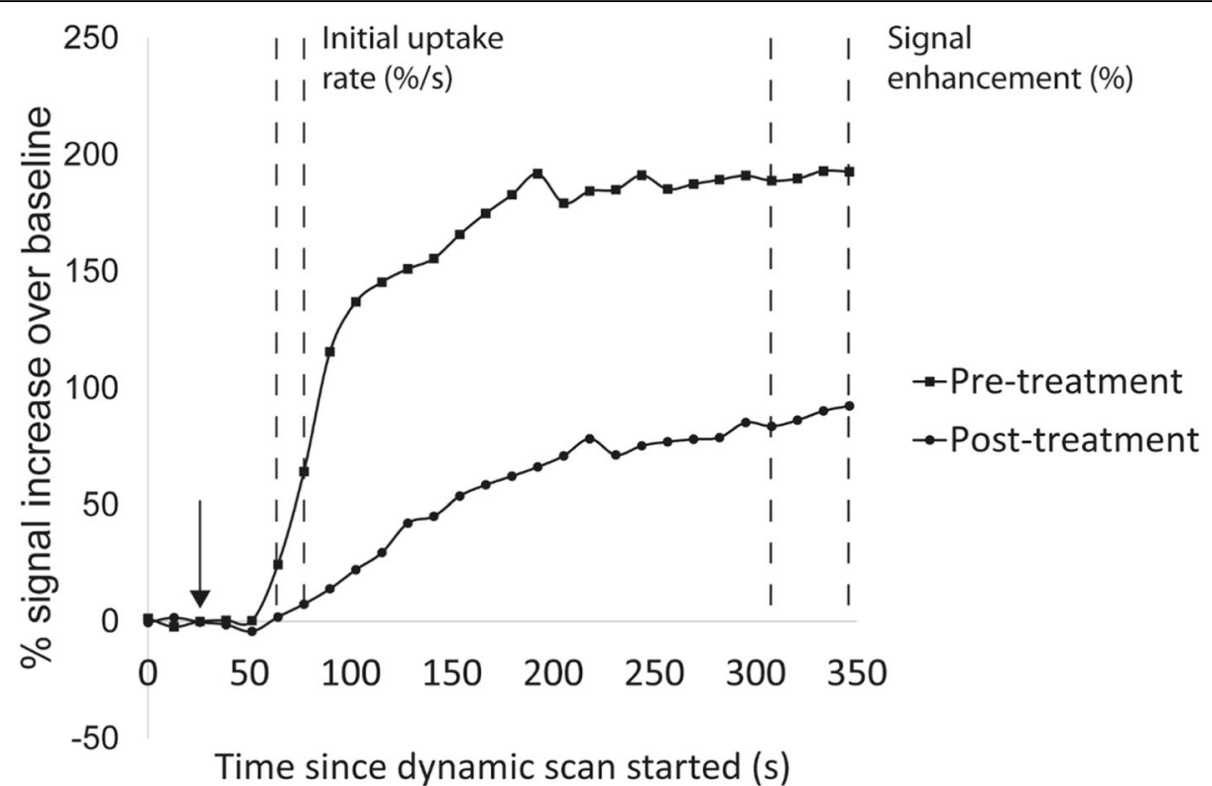

Fig. 2 Percentage gadolinium signal increase across the segmented synovial volume for subject 5. The contrast is injected at the time shown in the arrow. The initial uptake rate $(\% / \mathrm{s})$ is calculated from the gradient of the line of the first two positive signal increases and the signal enhancement (\%) is the mean of the last four points. This subject had a change in synovial volume from $72.2 \mathrm{~cm}^{3}$ pre-treatment to $0.7 \mathrm{~cm}^{3}$ posttreatment. The initial uptake shown reduces from $2.83 \% / \mathrm{s}$ to $0.51 \% / \mathrm{s}$ post-treatment and signal enhancement reduces from 190 to $90 \%$

research assessment was 14 weeks (range 12.6-19 weeks). The time required for subjects' MRI appointments is presented in the Additional file 1.

The median time (IQR) for contrast to reach the synovium after the beginning of the scan was 63 (14) seconds and contrast never reached synovium before the fifth image (range fifth-seventh image). Therefore, the uptake of gadolinium was followed for a median time of 315 (14) seconds after injection.

\section{Clinical assessments}

Baseline and follow-up clinical assessments are presented in Table 1 and Fig. 3. Clinician-assessed disease activity in the injected knee changed from present to absent in 7/10 participants post-IAGI. Two of the three participants with persisting clinician-assessed disease activity had a measurable synovial volume on follow-up MRI; one did not. All three participants with persisting clinically-assessed disease activity had repeat IAGI. This subsequently led to clinical improvement in the two with a measurable synovial volume and no improvement in the third young person. Although the AJC decreased significantly overall, it did not change in $4 / 10$ patients. The physician global score reduced after joint injection in all cases, as did the parent global score for 9/10 cases. Where the ESR was $>5 \mathrm{~mm}$ at baseline, it always decreased. 6/10 patients had an AJC of 0 following joint injection and 4 of these 6 patients had follow-up JADAS and cJADAS scores of less than or equal to 1 (consistent with inactive disease or remission): no other patients had JADAS/cJADAS scores indicating remission. Figure 3 illustrates that the parent global scores generally contributed more to the overall JADAS score than the AJC or physician global score with no suggestion of a relationship to ILAR subtype or disease duration.

\section{Qualitative MRI reports}

For the first MRI assessment, synovial thickening outside normal limits was reported for six of the subjects at baseline and reported within normal limits for five subjects (Tables 1 and 2). Minimal synovial enhancement in keeping within normal limits was noted in the report on two of the latter subjects, and no enhancement noted on the other three subjects. All reports for the second MRI assessment found no synovial thickening beyond normal limits. Chondral surfaces all appeared to be well maintained and there was no evidence of bone oedema.

\section{Quantitative MRI measurements}

The synovial volumes measured at baseline and at follow-up are given in Table 2. Across the 10 subjects who attended follow-up appointments the median (IQR) synovial volume was reduced from 38.5 (82.1) $\mathrm{cm}^{3}$ to $0.0(0.2) \mathrm{cm}^{3}(p=0.005)$. There were three signatures of synovial volume response. The first group $(n=6)$ had frank synovitis, called qualitatively by the radiologist as outside normal limits and with a median synovial volume of $79.6 \mathrm{~cm}^{3}\left(34.5 \mathrm{~cm}^{3}\right.$, Fig. 4). On post-treatment follow-up, these volumes reduced to a median of $0.0 \mathrm{~cm}^{3}\left(0.6 \mathrm{~cm}^{3}\right)$. The second group 


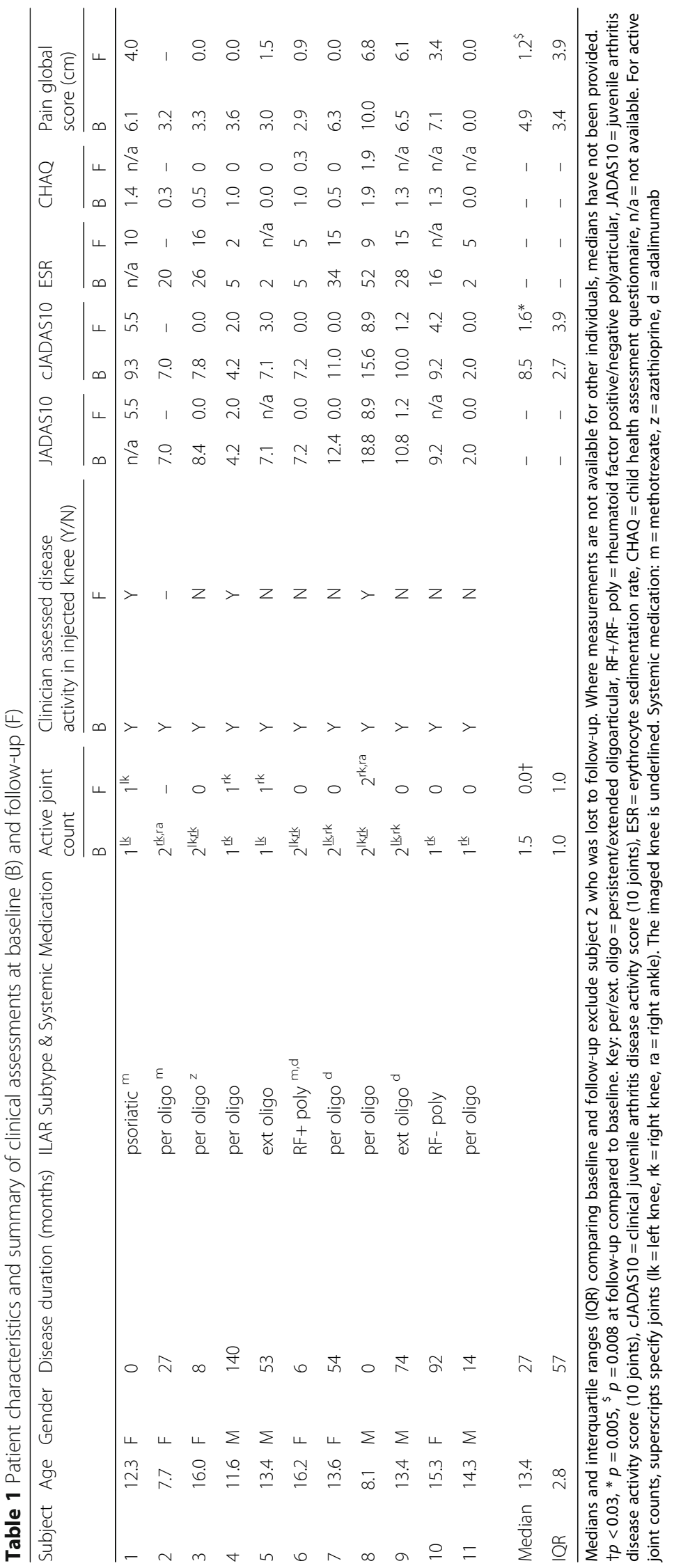




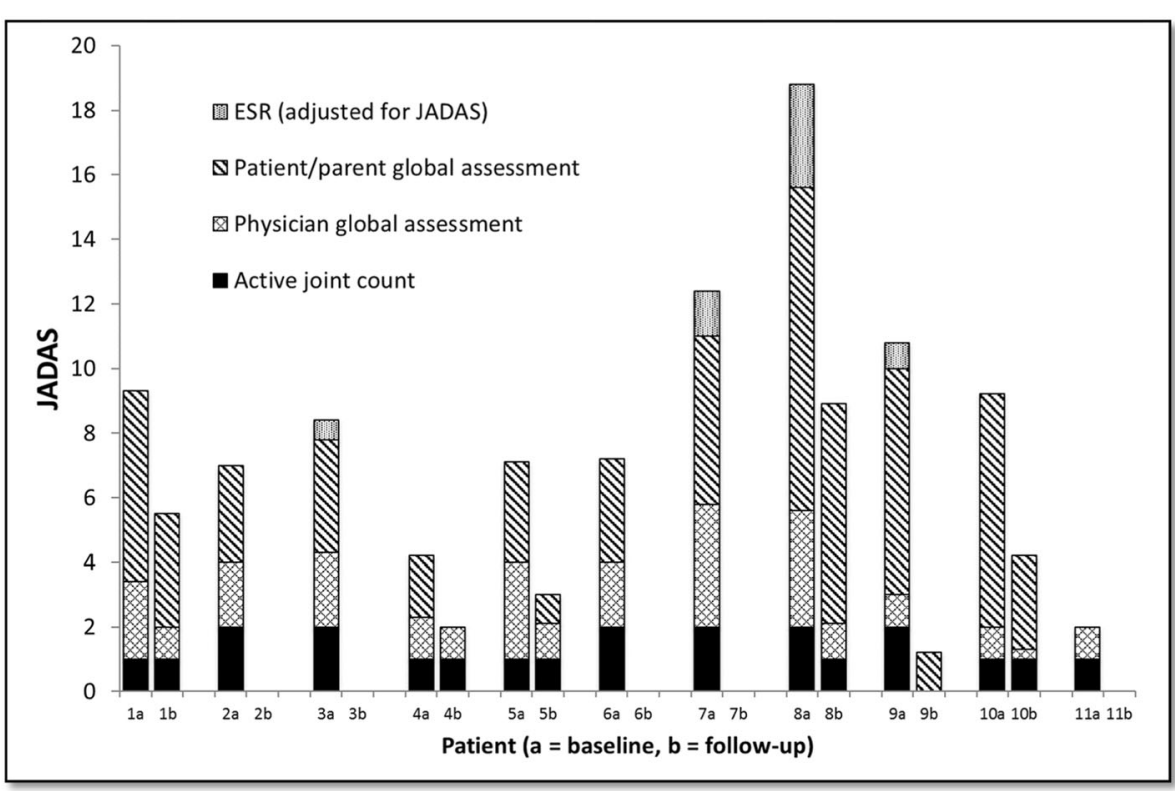

Fig. 3 The JADAS scores for the subjects at baseline and follow-up MRI scanning. Column shading indicates the relative contribution of the four JADAS components. The overall score is often dominated by the patient/parent global assessment, with no clear relationship to the physician assessment. There was no follow-up data available for subject 2, and blood tests were not clinically indicated for subject 1 at baseline and subjects 5 and 10 at follow-up. JADAS, juvenile arthritis disease activity score; IAGl, intra-articular glucocorticoid injection; ESR, erythrocyte sedimentation rate

$(n=3)$ had a measurable synovial enhancement (with volume $>1.0 \mathrm{~cm}^{3}$ ), were called within normal limits by the radiologist and had a median synovial volume of $1.2 \mathrm{~cm}^{3}\left(0.1 \mathrm{~cm}^{3}\right)$, which reduced to $0.0 \mathrm{~cm}^{3}\left(0.1 \mathrm{~cm}^{3}\right)$ post treatment. The remaining subject had a negligible initial volume which was unchanged posttreatment $\left(0.1 \mathrm{~cm}^{3}\right.$ vs. $\left.0.0 \mathrm{~cm}^{3}\right)$ within the limits of inter-observer reproducibility.

Both the median initial rate of contrast uptake and the median signal enhancement for the 10 young people

Table 2 Quantitative MRI results at baseline (B) and follow-up (F)

\begin{tabular}{|c|c|c|c|c|c|c|c|c|c|c|}
\hline \multirow[t]{2}{*}{ Subject } & \multicolumn{2}{|c|}{$\begin{array}{l}\text { Synovial volume } \\
\left(\mathrm{cm}^{3}\right)\end{array}$} & \multicolumn{2}{|c|}{$\begin{array}{l}\text { Maximum uptake } \\
\text { rate }(\% / s)\end{array}$} & \multicolumn{2}{|c|}{$\begin{array}{l}\text { Signal } \\
\text { enhancement (\%) }\end{array}$} & \multicolumn{2}{|c|}{$\begin{array}{l}\text { Radiologist reports synovial enhancement } \\
\text { beyond normal limits }(\mathrm{Y} / \mathrm{N})\end{array}$} & \multicolumn{2}{|c|}{$\begin{array}{l}\text { Clinician assessed disease activity } \\
\text { in injected knee }(\mathrm{Y} / \mathrm{N})\end{array}$} \\
\hline & B & $\mathrm{F}$ & B & $\mathrm{F}$ & B & $\mathrm{F}$ & B & $\mathrm{F}$ & $B$ & $F$ \\
\hline 1 & 25.7 & 0.0 & 1.5 & 0.0 & 116 & 0 & $Y$ & $N$ & $Y$ & $Y($ rpt IAGI) \\
\hline 2 & 0.6 & - & 0.5 & - & 35 & - & $\mathrm{N}$ & - & Y & - \\
\hline 3 & 1.2 & 0.0 & 0.4 & 0.0 & 57 & 0 & N & $N$ & Y & $\mathrm{N}$ \\
\hline 4 & 51.2 & 0.1 & 1.8 & 0.4 & 148 & 109 & Y & $N$ & Y & $Y($ rpt $|A G|)$ \\
\hline 5 & 72.2 & 0.7 & 2.8 & 0.5 & 190 & 90 & Y & $N$ & Y & $\mathrm{N}$ \\
\hline 6 & 0.1 & 0.0 & 0.0 & 0.0 & 10 & 6 & $\mathrm{~N}$ & $\mathrm{~N}$ & $Y$ & $\mathrm{~N}$ \\
\hline 7 & 92.2 & 0.0 & 6.7 & 0.0 & 225 & 0 & Y & $\mathrm{N}$ & Y & $\mathrm{N}$ \\
\hline 8 & 101.6 & 1.9 & 4.6 & 1.9 & 157 & 126 & Y & $N$ & Y & $Y($ rpt $|A G|)$ \\
\hline 9 & 87.0 & 0.0 & 7.1 & 0.0 & 228 & 0 & Y & $N$ & Y & $\mathrm{N}$ \\
\hline 10 & 1.2 & 0.0 & 0.6 & 0.0 & 95 & 0 & $\mathrm{~N}$ & $N$ & Y & $\mathrm{N}$ \\
\hline 11 & 1.1 & 0.2 & 1.5 & 0.8 & 145 & 103 & $\mathrm{~N}$ & N & Y & $N$ \\
\hline Median & 38.5 & $0.0 \dagger$ & 1.7 & $0.0^{\$}$ & 147 & $3+$ & - & - & & \\
\hline IQR & 82.1 & 0.2 & 3.3 & 0.5 & 82 & 100 & - & - & & \\
\hline
\end{tabular}

Baseline medians and IQRs (interquartile ranges) exclude subject 2 who was lost to follow-up. $+p=0.005,{ }^{\$} p=0.008$ at follow-up compared to baseline. Key: rpt $\mathrm{IAGI}=$ repeat intra-articular glucocorticoid injection after follow-up 


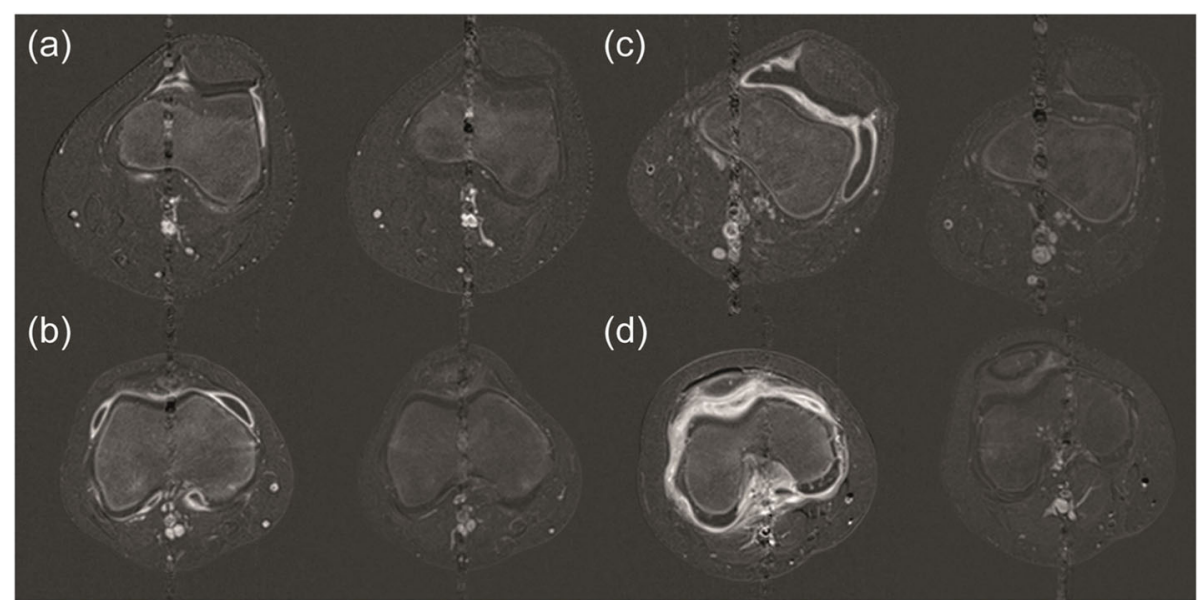

Fig. 4 Samples of difference images comparing measured synovial volume before and after IAGI. The difference images were derived from the pre- and post-contrast T1-weighted FS TSE images to highlight the contrast uptake in the synovium for four subjects: (a) 12 year-old female $\left(25.7 \mathrm{~cm}^{3}\right.$ vs. $\left.0.0 \mathrm{~cm}^{3}\right)$, (b) 11 year-old male $\left(51.2 \mathrm{~cm}^{3}\right.$ vs. $\left.0.1 \mathrm{~cm}^{3}\right)$, (c) 16 year-old female $\left(72.7 \mathrm{~cm}^{3} \mathrm{vs} .0 .7 \mathrm{~cm}^{3}\right)$ and $(\mathbf{d}) 8$ year-old male $\left(101.6 \mathrm{~cm}^{3} \mathrm{vs}\right.$. $\left.1.9 \mathrm{~cm}^{3}\right)$. The whole synovial volume is quoted in each case, not just for the slice shown. FS TSE, fat saturated turbo spin echo

completing were significantly reduced, from $1.7 \% / \mathrm{s}$ to $0.0 \% / \mathrm{s}, p=0.008$, and 147 to $3 \%, \mathrm{p}=0.005$ respectively (Table 2 and Additional file 1).

\section{Inter-observer agreement}

Bland-Altman analysis for inter-observer agreement for synovial volume showed no bias between observers $\left(-0.04 \mathrm{~cm}^{3}, 95 \%\right.$ limits of agreement $1.03 \mathrm{~cm}^{3}, \mathrm{p}=$ n.s. $)$, negligible compared to the median volume of $38.5 \mathrm{~cm}^{3}$. The bias and 95\% limits of agreement for the initial uptake rate of contrast were $0.01 \% / \mathrm{s}$ and $0.23 \% / \mathrm{s}$ respectively, while those for the signal enhancement were 2.7 and $12.9 \%$ respectively. The largest discrepancy in synovial volume between observers $\left(1.9 \mathrm{~cm}^{3}\right)$ occurred in the largest synovial volume measured $\left(101.6 \mathrm{~cm}^{3}\right)$. All other differences were below $0.6 \mathrm{~cm}^{3}$. Inter-observer differences are very small compared to the dynamic range of the intervention.

\section{Correlation between clinical and radiological outcomes}

There was a positive correlation between the pain global score and the initial synovial volume $(\rho=0.67, p<0.04)$. There was no significant correlation between the decrease in synovial volume post-treatment and the change in cJADAS, the change in global pain score, the parent global score or the change in active joint count (regardless of whether the total joint count or the joint count for the studied knee was used).

\section{Feedback from the questionnaire and telephone interview}

Feedback from the questionnaire and telephone interviews was positive; all subjects reported that they would recommend our research to their friends and families.
Five core experiential themes emerged from the data enabling identification of five discrete recommendations for paediatric MRI scanning (Table 3).

\section{Discussion}

We have demonstrated that quantitative MRI measurements can be used to quantify synovial disease activity in a stratified group of JIA patients undergoing IAGI. The positive subject feedback shows that it is both feasible and acceptable to collect quantitative MRI data in young people aged 7-16 years with new-onset knee synovitis.

Six subjects had synovial hypertrophy outside radiological standard limits at baseline with near-complete reduction in synovial volume at follow-up, an important observation for using quantitative MRI in the context of IAGI. Nine follow-up scans demonstrated significant reductions in synovial volume, the initial rate of contrast uptake and signal enhancement, demonstrating reduced vascularity of the remaining synovium.

In six cases with post-treatment follow-up, no enhancing synovial volume was detectable at follow-up while four subjects had a small but detectable volume. Five young people did not have synovial enhancement outside radiological reporting limits at baseline, similar to the $49 \%$ of clinically active patients who did not have synovitis according to JAMRIS classification in a qualitative cross-sectional study [36]. Van Gulik et al. reported normal MRI findings in $35 \%$ of patients with cliniciandefined knee synovitis [37]. In this study, quantitative MRI was able to detect volume change post IAGI in 3/4 baseline scans falling below the standard reporting threshold. 3/10 subjects had clinician-defined disease activity at follow-up, though the MRI were reported within 
Table 3 Themes and recommendations from the patient's perspective. Five core themes relating to the patient experience emerged from the data and from this we identified the following recommendations for paediatric MRI

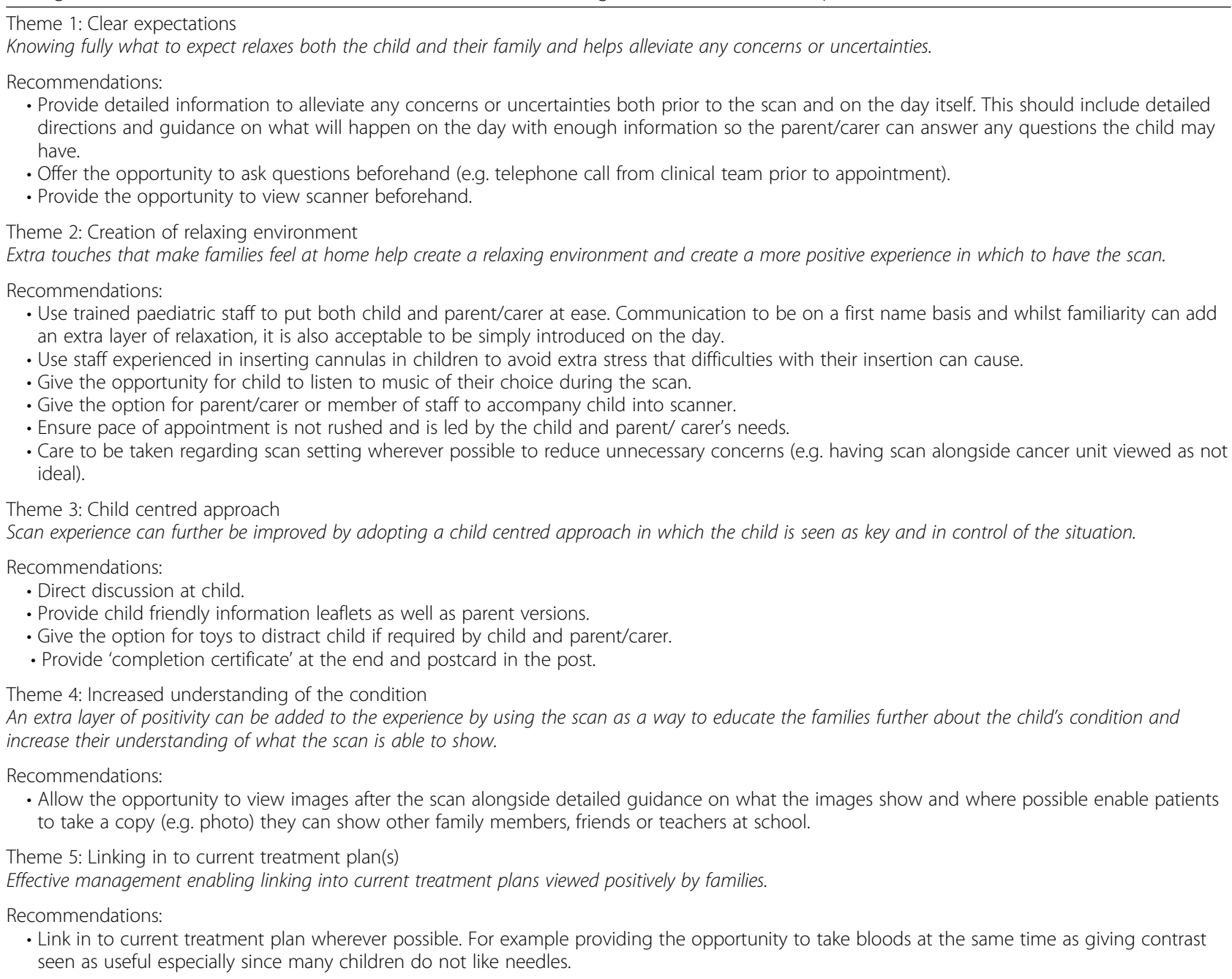

normal limits: there was a measurable synovial volume in two of these subjects. In retrospect, the negligible synovial volume for subject 6 suggests a non-synovial aetiology to their symptoms, despite the clinical response to IAGI.

Although the impact of IAGI on synovial volume and vascularity was frequently dramatic, the quantitative MRI changes were not always reflected in the composite clinical assessments. Of the six children with no detectable synovial volume post IAGI $\left(0.0 \mathrm{~cm}^{3}\right)$, only three would be regarded as in remission according to cJADAS $(</=1)$, whilst one child with a post-treatment cJADAS score of 0 had a measurable synovial volume. Our study adds further weight to a growing concern that the present definitions of remission may require reconsideration in light of future imaging data [14].

The post-joint injection clinical assessments illustrate that, in our cohort with low joint counts, the patient/ parent global scores contribute more to the JADAS score than the AJC/physician global score. This discrepancy between family / physician perception of impact of disease has been demonstrated previously [38] and our study further highlights the risk of underestimating the impact of oligoarthritis on everyday life.

There have been previous attempts to quantify synovial volume changes with treatment over similar timescales using quantitative MRI in JIA. Workie et al. studied the 3-month response to treatment, though only four subjects received IAGI at that time point [27], with modest synovial volume reduction of $26 \%$ and $29 \%$ in the initial uptake rate: the children recruited had a median of eight active joints at baseline.

A one-year follow-up of active wrist involvement in 36 children demonstrated a $50 \%$ reduction in synovial volume, though treatment types and durations were not specified [39]. In other arthritides, such as rheumatoid arthritis, more modest changes are reported with quantitative methods $[25,39,40]$. 
Our study suggests that quantitative MRI scanning adds important information both to clinical assessments and to current qualitative MRI reporting. We chose to assess the impact of IAGI in new onset knee synovitis, as knee synovitis is common. However, we anticipate that quantitative MRI reporting may have more potential in the assessment of disease activity in less accessible joints and in the presence of long-standing disease, joint damage or co-existing pain syndromes.

The limitations of our study include the small sample size studied. Although the study was open to sequential eligible families with young people aged 4-16 years, only one child younger than 7 years was approached. The family declined, citing concerns that their child may not lie still. Therefore, we cannot draw conclusions about the acceptability of MRI in the youngest children. Contact with one subject was lost before follow-up scanning, though their responses to the questionnaire and telephone interview following the first MRI visit were positive. The post-IAGI synovial volumes calculated in this study suggest that the synovium of healthy knee joints has a negligible volume on quantitative contrast-enhanced imaging. There are no quantitative contrast-enhanced data on young people with healthy knees: the closest study to date examined young people with knee pain and non-inflammatory diagnoses such as hypermobility and functional disorders [42].

The use of thresholding at defined signal enhancement levels reduced subjective choice between the analysts, with the decision dominating the analysis time (typically 20-30 min per volume) being that of separating the synovium from adjacent small blood vessels. These vessels appeared to have a consistent small volume on the pre- and post- IAGI imaging, so it may be possible to evaluate the volume much faster, perhaps three minutes, with further development of the analysis software and if the inclusion of minor vessels were to be tolerated, aiding clinical adoption. Translation to clinical practice requires careful selection of the management decisions in the clinical care pathway for which quantitative MRI would be used, since MRI availability is limited. These aspects are the subject of ongoing work.

This study focuses on the impact of IAGI in new-onset knee synovitis, measured by quantitative MRI. Whilst the near-complete resolution of knee synovitis post IAGI is an interesting observation, the true value of quantitative MRI techniques may be realised in future studies exploring the impact of current treatment regimes on less accessible joints such as the hip or temporomandibular joints and / or comparing the relative efficacies of different therapeutic regimes. Quantitative MRI has particular potential in young people with oligoarticular disease in whom clinical disease activity measures may not be sensitive or specific enough to reliably identify changes in the degree of localised synovial disease.

\section{Conclusions}

This study has demonstrated that quantitative MRI can measure the effect of IAGI on synovial volume and is accessible and feasible for children aged between 7 and 16 years, with positive feedback from families. Synovial volume was reduced markedly by IAGI, either to zero or close to zero. Quantitative MRI provided different information to traditional clinical disease activity measures and was able to identify more patients with an improved synovial volume than routine qualitative reporting. In summary, quantitative MRI has the potential to add important information to our understanding of disease outcomes in JIA.

\section{Supplementary information}

Supplementary information accompanies this paper at https://doi.org/10. 1186/s12969-019-0377-7.

Additional file 1. Supplementary Methods, Supplementary Results, Supplementary Fig. S1, Supplementary Fig. S2, Supplementary Table S1, Supplementary References.

\section{Abbreviations \\ CJADAS: three variable clinical juvenile arthritis disease activity score; CRP: C- reactive protein; ESR: Erythrocyte sedimentation rate; |AGI: Intra-articular glucocorticoid injection; ILAR: International League of Associations for Rheumatology; IQR: Interquartile range; JADAS: four variable juvenile arthritis disease activity score; JAMRIS: juvenile arthritis MRI scoring system; JIA: Juvenile idiopathic arthritis; MRI: Magnetic resonance imaging; VAS: Visual analogue scale}

\section{Acknowledgements}

The authors would like to thank Louise Fox from the Newcastle upon Tyne Hospitals NHS Foundation Trust for assistance in setting up our project and of the research radiographers Louise Jordan and Leoana McNamara from the Freeman Hospital, who helped to acquire the MRI data.

\section{Authors' contributions}

All named authors meet the International Committee of Medical Journal Editors (ICMJE) criteria for authorship for this manuscript, accept responsibility for the integrity of the work as a whole, and have provided final approval of the manuscript to be published.

\section{Funding}

The research costs and the support of JLB's research time was supported by the Medical Research Council, UK, by a Confidence in Concept award (MC) PC/15030), with support from the NIHR Clinical Research Network North East and North Cumbria.

\section{Availability of data and materials \\ The datasets used and/or analysed during the current study are available from the corresponding author on reasonable request, subject to the requirements of the study ethical approval and Caldicott approval.}

\section{Ethics approval and consent to participate}

The study, performed between September 2017 and August 2018, complied with the Declaration of Helsinki and obtained a favourable opinion from the Newcastle and North Tyneside 1 Research Ethics Committee (206363, 16/NE/ 0299) and the Health Research Authority, with the parents/carers of the subjects giving written informed consent, and written assent from the young people. 


\section{Consent for publication}

Consent for the publication of anonymised participant study results and images was obtained from all participants as part of the study consent process above.

\section{Competing interests}

The authors declare that they have no competing interests.

\section{Author details}

'Paediatric Rheumatology, Great North Children's Hospital, Newcastle upon Tyne Hospitals NHS Foundation Trust, Newcastle upon Tyne, UK. ${ }^{2}$ Musculoskeletal Research Group, Institute of Cellular Medicine, Newcastle University, Newcastle upon Tyne, UK. ${ }^{3}$ Paediatrics, Newcastle upon Tyne Hospitals NHS Foundation Trust, Newcastle upon Tyne, UK. ${ }^{4}$ Radiology, Freeman Hospital, Newcastle upon Tyne Hospitals NHS Foundation Trust, Newcastle upon Tyne, UK. ${ }^{5}$ Paediatric Radiology, British Columbia Children's Hospital, Vancouver, Canada. ${ }^{6}$ Newcastle Magnetic Resonance Centre, Institute of Cellular Medicine, Newcastle University, Newcastle upon Tyne, UK.

Received: 7 October 2019 Accepted: 4 November 2019

Published online: 21 November 2019

\section{References}

1. Petty RE, Southwood TR, Baum J, Bhettay E, Glass DN, Manners P, Maldonado-Cocco J, Suarez-Almazor M, Orozco-Alcala J, Prieur AM. Revision of the proposed classification criteria for juvenile idiopathic arthritis: Durban, 1997. J Rheumatol. 1998;25(10):1991-4.

2. Petty RE, Southwood TR, Manners P, Baum J, Glass DN, Goldenberg J, He X, Maldonado-Cocco J, Orozco-Alcala J, Prieur AM, Suarez-Almazor ME, Woo P. International league of associations for rheumatology classification of juvenile idiopathic arthritis: second revision, Edmonton, 2001. J Rheumatol. 2004:31(2):390-2.

3. Symmons DP, Jones M, Osborne J, Sills J, Southwood TR, Woo P. Pediatric rheumatology in the United Kingdom: data from the British pediatric rheumatology group National Diagnostic Register. J Rheumatol. 1996;23(11): 1975-80.

4. Foster HE, Marshall N, Myers A, Dunkley P, Griffiths ID. Outcome in adults with juvenile idiopathic arthritis: a quality of life study. Arthritis Rheum. 2003;48(3):767-75

5. Malviya A, Johnson-Lynn S, Avery P, Deehan D, Foster H. Juvenile idiopathic arthritis in adulthood and orthopaedic intervention. Clin Rheumatol. 2009; 28(12):1411-7

6. Beresford MW, Baildam EM. New advances in the management of juvenile idiopathic arthritis--2: the era of biologicals. Arch Dis Child Educ Pract Ed. 2009;94(5):151-6.

7. Coutinho AE, Chapman KE. The anti-inflammatory and immunosuppressive effects of glucocorticoids, recent developments and mechanistic insights. Mol Cell Endocrinol. 2011;335(1):2-13.

8. Ostergaard M, Halberg P. Intra-articular corticosteroids in arthritic disease: a guide to treatment. BioDrugs. 1998;9(2):95-103.

9. McErlane F, Beresford MW, Baildam EM, Thomson W, Hyrich KL. Recent developments in disease activity indices and outcome measures for juvenile idiopathic arthritis. Rheumatology (Oxford). 2013;52(11):1941-51.

10. Ruperto N, Ravelli A, Pistorio A, Malattia C, Cavuto S, Gado-West L, Tortorelli A, Landgraf JM, Singh G, Martini A. Cross-cultural adaptation and psychometric evaluation of the childhood health assessment questionnaire (CHAQ) and the child health questionnaire (CHQ) in 32 countries. Review of the general methodology. Clin Exp Rheumatol. 2001;19(4 Suppl 23):S1-9.

11. Guzman J, Burgos-Vargas R, Duarte-Salazar C, Gomez-Mora P. Reliability of the articular examination in children with juvenile rheumatoid arthritis: interobserver agreement and sources of disagreement. J Rheumatol. 1995: 22(12):2331-6.

12. Shoop-Worrall SJW, Kearsley-Fleet L, Thomson W, Verstappen SMM, Hyrich $\mathrm{KL}$. How common is remission in juvenile idiopathic arthritis: a systematic review. Semin Arthritis Rheum. 2017:47(3):331-7.

13. Shoop-Worrall SJW, Verstappen SMM, McDonagh JE, Baildam E, Chieng A, Davidson J, Foster H, loannou Y, McErlane F, Wedderburn LR, Thomson W, Hyrich KL. Long-term outcomes following achievement of clinically inactive disease in juvenile idiopathic arthritis: the importance of definition. Arthritis Rheumatol. 2018;70(9):1519-29.
14. Ravelli A, Consolaro A, Horneff G, Laxer RM, Lovell DJ, Wulffraat NM, Akikusa JD, Al-Mayouf SM, Anton J, Avcin T, Berard RA, Beresford MW, Burgos-Vargas R, Cimaz R, De Benedetti F, Demirkaya E, Foell D, Itoh Y, Lahdenne P, Morgan EM, Quartier P, Ruperto N, Russo R, Saad-Magalhaes C, Sawhney S, Scott C, Shenoi S, Swart JF, Uziel Y, Vastert SJ, Smolen JS. Treating juvenile idiopathic arthritis to target: recommendations of an international task force. Ann Rheum Dis. 2018;77(6):819-28.

15. Collado P, Vojinovic J, Nieto JC, Windschall D, Magni-Manzoni S, Bruyn GA, lagnocco A, D'Agostino MA, Naredo E. Toward standardized musculoskeletal ultrasound in pediatric rheumatology: Normal age-related ultrasound findings. Arthritis Care Res (Hoboken). 2016;68(3):348-56.

16. Roth J, Ravagnani V, Backhaus M, Balint P, Bruns A, Bruyn GA, Collado P, De la Cruz L, Guillaume-Czitrom S, Herlin T, Hernandez C, lagnocco A, Jousse-Joulin S, Lanni S, Lilleby V, Malattia C, Magni-Manzoni S, Modesto C, Rodriguez A, Nieto JC, Ohrndorf S, Rossi-Semerano L, Selvaag AM, Swen N, Ting TV Tzaribachev N, Vega-Fernandez P, Vojinovic J, Windschall D, D'Agostino MA, Naredo E. Preliminary Definitions for the Sonographic Features of Synovitis in Children. Arthritis Care Res (Hoboken). 2017;69(8):1217-23.

17. Ting TV, Vega-Fernandez P, Oberle EJ, De Ranieri D, Bukulmez H, Lin C, Moser D, Barrowman NJ, Zhao Y, Benham HM, Tasan L, Thatayatikom A, Roth J. Novel ultrasound image acquisition protocol and scoring system for the pediatric knee. Arthritis Care Res (Hoboken). 2019;71 (7):977-85.

18. Damasio MB, Malattia C, Martini A, Toma P. Synovial and inflammatory diseases in childhood: role of new imaging modalities in the assessment of patients with juvenile idiopathic arthritis. Pediatr Radiol. 2010;40(6):985-98.

19. Doria AS, Babyn PS, Feldman B. A critical appraisal of radiographic scoring systems for assessment of juvenile idiopathic arthritis. Pediatr Radiol. 2006; 36(8):759-72.

20. Malattia C, Consolaro A, Pederzoli S, Madeo A, Pistorio A, Mazzoni M, Mattiuz C, Magnano GM, Viola S, Buoncompagni A, Palmisani E, Hasija R, Ruperto N, Ravelli A, Martini A. MRI versus conventional measures of disease activity and structural damage in evaluating treatment efficacy in juvenile idiopathic arthritis. Ann Rheum Dis. 2013;72(3):363-8.

21. Miller E, Uleryk E, Doria AS. Evidence-based outcomes of studies addressing diagnostic accuracy of MRI of juvenile idiopathic arthritis. AJR Am J Roentgenol. 2009;192(5):1209-18.

22. Magni-Manzoni S, Malattia C, Lanni S, Ravelli A. Advances and challenges in imaging in juvenile idiopathic arthritis. Nat Rev Rheumatol. 2012;8(6):329-36.

23. Hemke $R$, van Rossum MA, van Veenendaal M, Terra MP, Deurloo EE, de Jonge MC, van den Berg JM, Dolman KM, Kuijpers TW, Maas M. Reliability and responsiveness of the juvenile arthritis MRI scoring (JAMRIS) system for the knee. Eur Radiol. 2013;23(4):1075-83.

24. Li M, Sauer A, Holl-Wieden A, Pabst T, Neubauer H. Diagnostic value of diffusion-weighted MRI for imaging synovitis in pediatric patients with inflammatory conditions of the knee joint. World J Pediatr. 2019. https://doi. org/10.1007/s12519-019-00232-8.

25. Fritz J, Galeczko EK, Schwenzer N, Fenchel M, Claussen CD, Carrino JA, Horger MS. Longitudinal changes in rheumatoid arthritis after rituximab administration assessed by quantitative and dynamic contrast-enhanced 3-T MR imaging: preliminary findings. Eur Radiol. 2009;19(9):2217-24.

26. Hodgson RJ, Barnes T, Connolly S, Eyes B, Campbell RS, Moots R. Changes underlying the dynamic contrast-enhanced MRI response to treatment in rheumatoid arthritis. Skelet Radiol. 2008;37(3):201-7.

27. Workie DW, Graham TB, Laor T, Rajagopal A, O'Brien KJ, Bommer WA, Racadio JM, Shire NJ, Dardzinski BJ. Quantitative MR characterization of disease activity in the knee in children with juvenile idiopathic arthritis: a longitudinal pilot study. Pediatr Radiol. 2007;37(6):535-43.

28. Giannini EH, Ruperto N, Ravelli A, Lovell DJ, Felson DT, Martini A. Preliminary definition of improvement in juvenile arthritis. Arthritis Rheum. 1997:40(7):1202-9.

29. Consolaro A, Ruperto N, Bazso A, Pistorio A, Magni-Manzoni S, Filocamo G, Malattia C, Viola S, Martini A, Ravelli A. Development and validation of a composite disease activity score for juvenile idiopathic arthritis. Arthritis Rheum. 2009:61(5):658-66.

30. McErlane F, Beresford MW, Baildam EM, Chieng SE, Davidson JE, Foster HE, Gardner-Medwin J, Lunt M, Wedderburn LR, Thomson W, Hyrich KL. Validity of a three-variable juvenile arthritis disease activity score in children with new-onset juvenile idiopathic arthritis. Ann Rheum Dis. 2013;72(12):1983-8.

31. Consolaro A, Bracciolini G, Ruperto N, Pistorio A, Magni-Manzoni S, Malattia C, Pederzoli S, Davi S, Martini A, Ravelli A. Remission, minimal disease 
activity, and acceptable symptom state in juvenile idiopathic arthritis: defining criteria based on the juvenile arthritis disease activity score. Arthritis Rheum. 2012;64(7):2366-74.

32. Consolaro A, Negro G, Chiara Gallo M, Bracciolini G, Ferrari C, Schiappapietra B, Pistorio A, Bovis F, Ruperto N, Martini A, Ravelli A. Defining criteria for disease activity states in nonsystemic juvenile idiopathic arthritis based on a three-variable juvenile arthritis disease activity score. Arthritis Care Res (Hoboken). 2014;66(11):1703-9.

33. Ostergaard M, Klarlund M. Importance of timing of post-contrast MRI in rheumatoid arthritis: what happens during the first 60 minutes after IV gadolinium-DTPA? Ann Rheum Dis. 2001;60(11):1050-4.

34. Family and Friends Test (FFT). https://www.nhs.uk/using-the-nhs/about-thenhs/friends-and-family-test-fft/. Accessed 14th June 2019.

35. Bland JM, Altman DG. Statistical methods for assessing agreement between two methods of clinical measurement. Lancet. 1986;1 (8476):307-10.

36. Hemke R, Maas M, van Veenendaal M, Dolman KM, van Rossum MA, van den Berg JM, Kuijpers TW. Contrast-enhanced MRI compared with the physical examination in the evaluation of disease activity in juvenile idiopathic arthritis. Eur Radiol. 2014;24(2):327-34.

37. van Gulik EC, Hemke R, Welsink-Karssies MM, Schonenberg-Meinema D, Dolman KM, Barendregt AM, Nusman CM, Maas M, Kuijpers TW, van den Berg JM. Normal MRI findings of the knee in patients with clinically active juvenile idiopathic arthritis. Eur J Radiol. 2018;102:36-40.

38. Sztajnbok F, Coronel-Martinez DL, Diaz-Maldonado A, Novarini C, Pistorio A, Viola S, Ruperto N, Buoncompagni A, Martini A, Ravelli A. Discordance between physician's and parent's global assessments in juvenile idiopathic arthritis. Rheumatology (Oxford). 2007;46(1):141-5.

39. Malattia C, Damasio MB, Basso C, Santoro M, Verri A, Pederzoli S, Mattiuz C, Viola S, Buoncompagni A, Madeo A, Mazzoni M, Rosendahl K, Lambot-Juhan K, Tanturri de Horatio L, Magnano GM, Ravelli A, Martini A. Novel automated system for magnetic resonance imaging quantification of the inflamed synovial membrane volume in patients with juvenile idiopathic arthritis. Arthritis Care Res (Hoboken). 2012;64(11):1657-64.

40. Hodgson RJ, O'Connor P, Moots R. MRI of rheumatoid arthritis image quantitation for the assessment of disease activity, progression and response to therapy. Rheumatology (Oxford). 2008;47(1):13-21.

41. Tam LS, Griffith JF, Yu AB, Li TK, Li EK. Rapid improvement in rheumatoid arthritis patients on combination of methotrexate and infliximab: clinical and magnetic resonance imaging evaluation. Clin Rheumatol. 2007;26(6):941-6.

42. Hemke R, van den Berg JM, Nusman CM, van Gulik EC, Barendregt AM, Schonenberg-Meinema D, Dolman KM, Kuijpers TW, Maas M. Contrastenhanced MRl findings of the knee in healthy children; establishing normal values. Eur Radiol. 2018;28(3):1167-74.

\section{Publisher's Note}

Springer Nature remains neutral with regard to jurisdictional claims in published maps and institutional affiliations.

Ready to submit your research? Choose BMC and benefit from:

- fast, convenient online submission

- thorough peer review by experienced researchers in your field

- rapid publication on acceptance

- support for research data, including large and complex data types

- gold Open Access which fosters wider collaboration and increased citations

- maximum visibility for your research: over $100 \mathrm{M}$ website views per year

At BMC, research is always in progress.

Learn more biomedcentral.com/submissions 